\title{
Functional Porous Materials via Sol-Gel with Phase Separation
}

\author{
Kazuki NAKANISHI \\ Department of Chemistry, Graduate School of Science, Kyoto University, Kitashirakawa, Sakyo-ku, Kyoto-shi 606-8502
}

\begin{abstract}
Variety of functional porous materials in metal oxide, metalloxane polymer and even pure organic polymer compositions obtained by a sol-gel process are reviewed. Specifically, processes including polymerization-induced phase separation have been extensively introduced from theories to practices. The basic concept of hierarchical pore architecture in monolithic materials is explained, together with several examples of successful applications. Depending on the hierarchy, single, double and triple, unique family of materials can be fabricated. Existing and prospective applications and future challenges of the materials are also shown.
\end{abstract}

[Received December 1, 2006]

Key-words : Sol-gel, Phase separation, Spinodal decomposition, Porous materials, Oxides, Organic-inorganic hybrids, HPLC columns

Introduction

$\mathbf{S}_{\mathrm{s}}^{\mathrm{I}}$ NCE the "discovery" of the phase separation in gelling silica system, nearly twenty years have passed. Among wide-spread possibilities of sol-gel materials, thin films or coatings have been, and they still are, regarded as most promising form of final products. Dense and optically transparent materials have been pursued in the forms of thin film, fiber and even bulk. The regulation of porous properties in a gel, an intermediate product, was studied in order to efficiently eliminate the porosity in the final sintering stage. Sol-gel oxide materials characterized by the well-defined macropores were out of any existing category of inorganic gels. They were, however, very similar in morphology to the porous glasses prepared via metastable phase separation in immiscible glass-forming oxide systems followed by selective leaching of one of the separated phases. ${ }^{1)}$ The overall dynamics of phase separation and sol-gel transition in silica system was found to more resemble those observed in organic polymer systems. ${ }^{2)}$ Regarding the morphology formation process as a competition between dynamics of polymerization-induced phase separation and of steep chemical sol-gel transition, dependence of macroporous morphology on various synthesis parameters has been consistently interpreted..$^{3), 4)}$ The experimental system has been readily extended to organo-silicon and silica-based multicomponent oxide systems. ${ }^{5)}$ It has been found only recently, however, pure titanium, zirconium and aluminum oxides with well-defined macropores can also be fabricated. $\left.{ }^{6)}, 7\right)$ In the course of discovering oxide gel systems which can be controlled favorably for concurrent phase-separation and sol-gel transition, it has been found that the kind of gel-forming reaction, whether it is chemical polymerization, colloidal aggregation or oxolation of hydroxylated species, has a minor effect on the structure-formation process. ${ }^{8)}$ Phase separation phenomena related to the heterogeneity formation in materials have been investigated first in metallic alloys and oxide glasses, ${ }^{9)}$ followed extensively by polymeric materials ${ }^{10)}$ mainly due to their slow dynamics of developing heterogeneity. In biphasic materials with co-continuous (or bicontinuous) domains, porous materials can be obtained by the selective extraction of either of the phases. An industrial process of fabricating welldefined porous silica-rich glasses has been established by Corning Glass Works about a half century ago. ${ }^{1)}$ Because the process involved dissolution of alkaline-borate glass phase out of the pre-formed phase-separated glass, it required a relatively long processing time depending on the thickness of the product. By contrast, the chemically-polymerized phase-sepa- rated structure form a solid gel phase and an easily removable fluid phase. The simpler and quicker processing of gel-derived porous materials contributed a lot to commercialize the resultant hierarchically porous silica monolith as the separation media for high performance liquid chromatography, HPLC. ${ }^{11)}$ In the present review, a polymerization-induced phase separation accompanied by a sol-gel transition is described, taking polysiloxane systems as major examples.

\section{Background and principles}

Starting from the systems containing tetraalkoxysilane and appropriate additives, the polymerization-induced phase separation, especially the spinodal decomposition, has been extensively utilized to generate well-defined heterogeneous structures. ${ }^{5)}$ The most characteristic morphology of the biphasic spinodal decomposition is a co-continuous structure where both phase domains are continuous and mutually interconnected. Since only one of the phases forms three-dimensional network to solidify the whole system, the other fluidic phase can easily be removed to leave pore spaces in the length scale of the developed phase domains. A specific point that the polymerization-induced phase separation distinguishes itself from the thermally-induced phase separation in many metastable glass or polymer systems is its irreversible nature of chemical bond formation. In the case of thermally induced phase separation, the dynamics of the development of phase domains can be externally controlled by the temperature. One can quench the shape and size of the developing phase domains simply by decreasing the temperature of the system, which usually accompanies viscosity increase and liquid-toglass transition, thus arresting the transient structures at an arbitrary stage of the dynamics. On the other hand, the structure formation mechanism has more or less spontaneous nature in a system which undergoes chemically induced phase separation paralleled with crosslinking reaction. Both the onset of phase separation and sol-gel transition by crosslinking are governed by the kinetics of chemical bond formation. With a pre-determined starting composition and reaction temperature, the solution is just left to react at a constant temperature and in a closed condition (to avoid evaporation of volatile components). A sharp transition from sol to gel by an irreversible bond formation is essential in quenching the dynamically developing phase domains as a three-dimensional snap-shot. External regulations such as temperature change usually result in deteriorated final structures due to a complicated effect on the local structure forming dynamics. In 
Table 1. Examples of Starting Compositions which Exhibit Concurrent Phase Separation and Sol-Gel Transition

\begin{tabular}{|c|c|c|c|c|c|}
\hline Precursors & $\begin{array}{l}\text { Hydrolysis/Polycondensation } \\
\text { Conditions (mol } / \mathrm{mol} \text { ) }\end{array}$ & Additives (excl. Water, Catal.) & Gel Phase & Fluid Phase & References \\
\hline Tetraalkoxysilane & Water $/ \mathrm{Si}<2$ & Polar Solvent & Siloxane & Solvent Mixture & 2 \\
\hline Alkyltrialkoxysilane & Water $/ \mathrm{Si}<3$ & Alcohols & Alkyl-terminated Siloxane & Solvent Mixture & 16 \\
\hline Bis(trialkoxysilyl)alkane & Water $/ \mathrm{Si}>10$ & None & Alkylene-bridged Siloxane & Solvent Mixture & $17,32,33$ \\
\hline Tetraalkoxysilane & Water $/ \mathrm{Si}>4$ & $\begin{array}{l}\text { Strongly Hydrogen-bonding } \\
\text { Polymer, Nonionic Surfactant, } \\
\text { Cationic Surfactant }\end{array}$ & Siloxane + Polymer & Solvent Mixture & $15,29,30,31$ \\
\hline Tetraalkoxysilane & Water $/ \mathrm{Si}>4$ & $\begin{array}{l}\text { Weakly Hydrogen-bonding } \\
\text { Polymer, Anionic Surfactant }\end{array}$ & Siloxane & Solvent Mixture + Polymer & 3,4 \\
\hline Water Glass & Destabilization by acid & $\begin{array}{l}\text { Weakly Hydrogen-bonding } \\
\text { Polymer } \\
\text { Anionic Surfactant }\end{array}$ & Polysilicate & Sovent Miture + Polymer & $20,21,22,23$ \\
\hline Titania Colloid & Destabilization by base & $\begin{array}{l}\text { Strongly Hydrogen-bonding } \\
\text { Polymer }\end{array}$ & Titanoxane + Polymer & Solvent Mixture & 6 \\
\hline Titanium Alkoxide & Water $/ \mathrm{Si}>4$ & & Titanoxane + Polymer & Solvent Mixture & 7 \\
\hline Zirconium Alkoxide & Water $/ \mathrm{Si}>4$ & & Zirconoxane + Polymer & Solvent Mixture & Unpublished \\
\hline Aluminum Chloride & Water $/ \mathrm{Si}>4$ & $\begin{array}{l}\text { Strongly Hydrogen-bonding } \\
\text { Polymer }\end{array}$ & Siloxane & Solvent Mixture + Polymer & 18 \\
\hline
\end{tabular}

Table 1, the experimental systems that have been confirmed to give well-defined macroporous structures due to polymerization-induced phase separation and sol-gel transition are summarized. It is noteworthy that not a few gel-forming systems exhibit common features of phase separation and sol-gel transition, although the origin of gel-forming reaction is substantially different from each other. Below, important principles and phenomena related to the polymerization-induced phase separation in chemically crosslinking system are described in some more details.

\subsection{Polymerization-induced phase separation}

In most so-called "sol-gel processing" of various metal oxides, metal alkoxides are popular starting materials. The hydrolysis and polycondensation behaviors of silicon alkoxides have been extensively studied because of their exceptionally slow reaction kinetics and, therefore, the ease of obtaining homogeneous-looking gels under ambient and controlled conditions. ${ }^{12)}$ Oxide gels can be prepared from colloidal particles dispersed in an appropriate fluid medium (usually an aqueous medium) by destabilizing the dispersion. Metal salts which can be hydrolyzed and polycondensed can also be used as precursors using aqueous medium as a solvent. In the following theoretical part of the polymerization-induced phase separation, we in principle limit ourselves to chemical polymerization reactions, represented by polycondensation reactions of hydrolyzed metal alkoxides.

Let us first consider a typical hydrolysis-polycondensation of alkoxysilanes under acidic conditions, which gives a relatively narrow molecular weight distribution of the polymerizing oligomers. ${ }^{12)}$ The average molecular weight of the polymerizing species in a solution increases with reaction time by virtually irreversible polycondensation reactions among the monomers/oligomers. The thermodynamics of a solution containing polymerizing species tells us that mutual solubility among the constituents becomes lower as the average molecular weight of the polymerizing species increases. ${ }^{13)}$ This is mainly due to the loss of entropy of mixing among the constituents, which leads to the increase of the free energy of mixing, $\Delta G$.

$$
\Delta G=\Delta H-T \Delta S
$$

The reduction in mutual solubility caused by polymerization is contrasted with that caused by physical cooling of the system. ${ }^{14)}$ In the latter case, the free energy of mixing is increased by lowering the temperature. In both cases, a multicomponent system becomes less stable as the absolute value of the $T \Delta S$ term decreases. In some cases, changes in the polarity of oligomers with the generation and/or consumption of silanol groups may contribute to increase the $\Delta H$ term, which will also destabilize the system against homogeneous mixing. In any case, when the sign of $\Delta G$ becomes positive, the thermodynamic driving force for phase separation is generated in the system. In real experimental systems, poor solvents of the oligomers, several kinds of water-soluble polymers and surfactants can be used as an additive component to induce the phase separation in the course of a sol-gel reaction as summarized in Table 1.

1.2 Morphology development by spinodal decomposition

In a phase diagram with a miscibility window, the twophase region is divided into two sub-regions. One is that between binodal and spinodal, called the metastable region. In the metastable region, any infinitesimal fluctuation of the composition is energy-consuming, that is, even under the positive driving force to phase separation, finite activation energy is required to develop phase-separated domains. The typical phase separation mechanism in this region is the "nucleation and growth" where dispersed small regions called "nuclei" grow accompanied by an addition of constituents diffusing from the bulk (not yet separated) regions of the system. The natural consequence of this mechanism is a morphology with "dispersed A" and "matrix B" phase domains. The other region is that within a spinodal line, called the unstable region. In the unstable region, any infinitesimal fluctuation energetically stabilizes the system so that the fluctuation spontaneously develops with time without requiring activation energy. Depending mainly on the depth of quench (the difference between the critical temperature and the actually quenched temperature) and the mobility of the constituents (more pre- 
cisely, that of diffusing units), only a single Fourier component among the various fluctuation wavelengths survives. After a long enough elapse of time, this component dominates the characteristic size of the phase domains in the initial stage of phase separation..$^{9)}$ One clear difference that can be seen between the nucleation and growth mechanism is that the phase domains have no distinct interface in the initial stages of the phase separation. The contrast in chemical composition develops continuously with time until the equilibrium phase compositions are reached. Under comparable volume fractions of conjugate phase domains without crystallographic or mechanical anisotropy, a sponge-like structure called a co-continuous structure forms. The co-continuous structure is characterized by mutually continuous conjugate domains and hyperbolic interfaces.

In the classical description of the initial stage of spinodal decomposition, the co-continuous structures are reproduced by the superposition of a number of sinusoidal compositional waves. ${ }^{9)}$ The real structure development, however, strongly depends on the dynamics driven by the interfacial energy. ${ }^{10}$ With an increase of concentration difference between the conjugate phase domains, the interfacial energy piles up. In order to reduce the total interfacial energy, the system reorganizes the domain structure toward that with less interfacial area and less local surface energy arising from the curvature distribution. The reduction of total interfacial area can be achieved by coarsening the structure. The self-similar coarsening of the spinodally phase-separated domains is well known in many oxide glass or organic polymer systems. Within the regime of self-similar coarsening, the geometrical features of the separating domains remain unchanged except the characteristic size. The coarsening is followed and often overwhelmed by the fragmentation of the continuous domains. Since the co-continuous structure of spinodal decomposition is consisted of both negative and positive curvature surfaces, a curvature of either sign should be eliminated to attain the most stable interfacial configuration. This local surface-energetic requirement drives the fragmentation of either of the conjugate phases. Consequently, the well-defined co-continuous structure of the spinodal decomposition is a transient one, which coarsens self-similarly for a limited duration of time and then breaks up into fragmented structures.

\subsection{Structure freezing by sol-gel transition}

Sol-gel transition is a dynamic phenomenon which strongly depends on the network connectivity. In the case of virtually irreversible chemical sol-gel transition in a solution, a constant increase in the degree of polymerization of individual clusters contributes to increase the solution viscosity only gradually in the earlier stage of polymerization. The continued growth of clusters, however, reaches a point where the first single connection across the system dimension is formed. Around this point, typically denoted as the sol-gel transition point, the steep increase and divergence of viscosity is observed. As a result, the system turns from a viscous fluid to an elastic solid. Due to the continued polymerization reactions within the loosely connected network, the network density gradually increases to arrest motions of constituents in finer length scales.

If any transient (dynamic) heterogeneity is present in a gelling solution, it will be arrested in the gel network when the timescale of the sol-gel transition is short enough to freeze the "snap-shot" structure of the transient heterogeneity. In the sol-gel system based on an acid-catalyzed hydrolysis/polycondensation of alkoxysilane, both the structural evolution due to phase separation and the structure freezing by sol-gel transi- tion take place as a result of irreversible polycondensation reactions. The "frozen" structure depends, therefore, on the onset of phase separation relative to the "freezing" point by sol-gel transition. The earlier the phase separation is initiated relative to the sol-gel transition, the coarser the resultant structure becomes, and vice versa.

Among a lot of reaction parameters of a sol-gel reaction, those that strongly influence the mutual solubility of the constituents and/or the hydrolysis/polycondensation reaction kinetics play important roles in determining the final size of the phase separated domains in the gelled specimens. For example, a higher reaction temperature normally increases the mutual solubility of the constituents and hence suppresses the phase separation, and in parallel it accelerates the hydrolysis/ polycondensation reactions. Due to these duplicate effects, gels with finer phase separated domains are obtained at higher temperatures. If one adds a co-solvent of the relatively incompatible components in the reaction solution, the phase separation is suppressed accompanied usually by decreased hydrolysis/polycondensation reaction rates due to the dilution effect. In this case, the resultant morphology depends on the competitive effects of suppressed phase separations and decelerated hydrolysis/polycondensation reactions.

Another important parameter which determines the gel morphology is the relative volume fraction of the "fluid" phase which converts to macropores after drying. With a few exceptions, the main component of "fluid" phase is usually a solvent mixture. The volume fraction of the solvent then becomes the crucial parameter in determining the pore volume and overall connectivity of the gel skeletons in the resultant gel structure. With an appropriate choice of these reaction parameters, the pore size (domain size) and pore volume of the gels can be designed in a wide variety of morphologies.

\section{Examples of materials with contolled macropores}

\subsection{Pure silica}

As extensively listed in Table 1, pure silica formulations have been exploited to give every possible morphology, material shape and doped compositions. ${ }^{5)}$ In the presence of limited amount of water, using especially tetramehoxysilane (TMOS) as a precursor, the phase separation is induced just by adding polar solvent. ${ }^{2}$ In the presence of higher molar ratio of water to silicon, the phase separation is induced by polymeric or amphiphilic additives. ${ }^{3), 4), 5), 8), 15)}$ Polymers or surfactants having no specific attractive interaction with silanol surfaces, e.g. poly (acrylic acid) or anionic polymers and surfactants, tend to be distributed to the "fluid phase," so that the amount of additives directly relates to the volume fraction of macropores. On the other hand, due to the strong hydrogen-bonds between silanols and polyoxyethylenes, additives having $-\mathrm{CH}_{2}-\mathrm{CH}_{2}-\mathrm{O}-$ repeating units, poly (ethylene oxide) (PEO) and Pluronic or Brij families, are always distributed to the "gel-phase," while the solvent mixture becomes a majority in the "fluid phase." Similarly, cationic surfactants are preferentially distributed to the "gel-phase." In these cases, the volume fraction of the "fluid phase" can be controlled mostly by the amount of solvent, while the domain size is determined by the additive concentration which dominantly governs the phase separation tendency. This implies that one can independently design the volume and size of macropores by the concentrations of solvent and that of additive, respectively. In addition, the strong attractive interaction between silica and additive molecule makes it possible to template the mesoscale structures of the structural unit of the gels by surfactants as described below. 


\subsection{Siloxane-based organic-inorganic hybrids}

Among the silicon alkoxides having trialkoxysilyl ligands, clear distinction can be made between alkyltrialkoxysilanes, represented by methyltrimethoxysilane (MTMS) and bis (trialkoxysilyl)-alkanes, represented by bis (trimethoxysilyl) ethane (BTME).$^{16), 17)}$ Due to high tendency of forming cyclic species in their polycondensation stage, gels obtained in an MTMS system often retain high density of methyl groups on the surface of the oligomers. On the other hand, gels prepared in a BTME system exhibit apparently higher surface hydrophilicity comparable to that of pure silica gels probably because ethylene bridges between the silicon atoms are buried in the network rather than exposed on the surface of the oligomers. It is also experimentally confirmed that MTMSderived oligomers phase-separate in relatively less polar solvent composition, however, BTME hardly phase-separates even in highly polar solvent compositions. ${ }^{9)}$ Due to the presence of Si-C bonds, gels from both MTMS and BTME precursors exhibit much higher alkaline resistance relative to pure silica gels. Similar to the pure silica case, the BTMEderived gels with silanol-rich surfaces can be templated by surfactants to exhibit mesopores with well-defined size and shape.

\subsection{Titania and zirconia}

Alkoxides of titanium and zirconium generally exhibit much higher reactivity toward hydrolysis/polycondensation than those of silicon, mainly due to the difference in partial charge of the metals in their respective oxygen-coordinated environments. Well-defined macroporous titania and zirconia have been very hard to be synthesized in monolithic form. Very recently, Konishi et al. reported that the use of titanium $n$ propoxide instead of $i$-propoxide under high concentration of hydrochloric acid enabled one to control the hydrolysis/polycondensation kinetics in an experimentally feasible time scale, where gelation occurs in a few tens minutes to a few hours. ${ }^{7}$ The reaction can be well-controlled with relatively limited amount of water against complete hydrolysis, so that titania oligomers rich in unreacted alkoxy groups separates from the solvent mixture containing highly polar compound such as formamide. Incorporation of an appropriate amount of PEO is effective for an adjustment of phase separation dynamics to obtain better defined macroporous structures. Macroporous titania gels thus obtained are composed of microcrystalline anatase phase even in the wet-gel stage. By controlling the growth of the anatase crystallites during the aging and heattreatment, the interstices of the crystallites form sharply distributed mesopores in the size range of $5-10 \mathrm{~nm}$. Monolithic pure zirconia with controlled macropores can also be prepared with further cares on synthesis. Zirconia tends to form gels with amorphous structural units and transforms via monoclinic into tetragonal as the heat-treatment temperature increases.

\subsection{Alumina}

Water-soluble metal salts are low-cost precursors and are available in broader range of metallic elements compared with alkoxides. The drawback of using metallic salt is that most of them are too ionic to be fabricated into amorphous-like threedimensional networks (analogous to the "network modifiers" in oxide glass compositions). In this respect, trivalent smallsized ions such as boron and aluminum, actually their hydroxylated complexes, can be possible candidates to be used in the synthesis of monolithic gels. Based on the methods reported on alumina, iron oxide and many others by Gash et al., the polymerization-induced phase separation has been successfully combined with the gel-forming reaction assisted by the ringopening reaction of propylene oxide. ${ }^{18)}$ An addition of high molecular weight (MW $>100 \mathrm{kDa}$ ) of PEO was again found to be effective to slow down and precisely regulate the phase separation dynamics. The added PEO is mainly distributed to the fluid-phase and has minor effect on the mesopore formation. Macroporous morphologies and pore size distributions of $\alpha$-alumina ceramics thus obtained are very similar to those of silica with weakly-interacting polymers. ${ }^{3), 4)}$ On heat-treatment, the monolithic gels transformed from amorphous via $\gamma$-alumina to $\alpha$-alumina crystalline form without damaging the macroporous framework.

\subsection{Oxides from colloidal dispersion and polysilicate} solution

Colloidal dispersion of oxide particles and polyoxometallate solution are another category of conventional precursors of gel materials. An efficient process to control macroporous structure in alkaline silicate - colloidal silica mixtures has been established by Shoup et al. ${ }^{19)}$ The gelation of alkaline silicate (so called water-glass) was modified by the colloidal particles to give continuous macropores up to sub-micrometers in diameter. Due to the inclusion of a considerable amount of rigid particles in a gel, as well as to the presence of continuous macropores which efficiently transport the solvent liquid, the drying shrinkage of the gel was effectively suppressed to avoid cracking and fracture. High silica glass products can be manufactured through the process by molding the gelling solution into various shapes.

Using alkaline silicate solution as an only silica source, Takahashi and co-workers has demonstrated that phase-separation can be induced in the gelation process by acidifying the solution. ${ }^{20)-23)}$ Well-defined co-continuous macroporous structures have been successfully controlled using water-soluble polymers such as poly (acrylic) acid, poly (vinyl alcohol) or anionic surfactant such as sodium dodecylsulfonate. The gelation of alkaline silicate solution occurs mostly by polycondensation between molecularly dissolved anionic silicate species, giving higher density of 4-fold coordinated silicon compared with that observed in alkoxy-derived systems. ${ }^{24}$ Although an extensive washing process is required to eliminate excess alkaline components, the porous gels are mechanically stable so that the water-glass based process is favored for large-scale production.

Konishi et al. tried to solidify an aqueous dispersion of commercially available fine colloidal anatase while inducing the phase separation in the course of gelation. ${ }^{6}$ ) By adding formamide to an acid-stabilized colloidal anatase dispersion at elevated temperature, the $\mathrm{pH}$ of the dispersion was increased gradually and homogeneously by the hydrolysis of formamide. With an incorporation of high molecular weight PEO (MW $>100 \mathrm{kDa}$ ), found were the well-defined macroporous morphology in resultant monolithic gels. Results also revealed that added PEO preferentially distributed to the gelphase and that the continuity of the gel skeletons improved with an increase of molecular weight of incorporated PEO. The colloidal route to macroporous oxide gels can be a versatile method since various kinds of useful oxide dispersions will be prepared into well-defined macroporous assemblages. The mechanical strength of the materials, however, has much to be improved since the gel network is composed of physically aggregated particles exhibiting compressive strength values about two orders lower than those for alkoxy-derived counterparts.

The common features of polymerization-induced phase separation in this last category of precursors compared to those in metal alkoxides indicates that any kind of polymerization to form three-dimensional network can equally induce 
spinodal decomposition only if an appropriate conditions is given as discussed in the literature. ${ }^{8)}$

\subsection{Organic polymer}

Based on the above common principle, fully organic polymer systems that form three dimensional crosslinked network and well-defined macropores have been explored by Kanamori et al. ${ }^{25)}$ Porous polymer beads have long history as a packing material of HPLC column. Styrene monomers (2-functional) are radically polymerized in the co-presence of divinylbenzene (4-functional) with additions of low-molecular weight additives called "porogen." With the development of crosslinked oligomers in the reaction system, they become incompatible with the solvent phase containing porogens, and a microphase separation occurs to generate sub-micrometer scale heterogeneity. The pore size distribution, however, is broad extending the distribution tail to the smaller pore size due to the fact that the network is composed of randomly aggregated globular structural units. Adopting a living radical polymerization condition together with an addition of counter polymer instead of low molecular weight porogen, monolithic pure poly(divinylbenzene) polymer having well-defined macropores has been prepared. The pore size and pore volume can be precisely controlled by reaction conditions. Due to highly continuous smooth structural units (skeletons), measured mechanical strength was comparable to that of macroporous glass with fully dense skeletons. Since carbon and other nonoxide ceramics are recently synthesized from polymeric precursors, polymeric monolith with controlled pores might become a special type of precursor body to such non-oxide ceramic products.

\section{Mesopore control and hierarchical pore structures}

\subsection{Single hierarchy}

Using the above process of designing macropore structures, a single hierarchy of either macropore or mesopore can be fabricated in monolithic materials. Materials having solely co-continuous macropores are prepared by either fully sintering the gel skeletons ${ }^{26)}$ or synthesizing virtually nonporous (without smaller pores) skeletons in organic-inorganic hybrid compositions. ${ }^{16)}$ For a kind of filtration purposes, gel (or glass) skeletons having no additional adsorption capacity is favored. In addition, mechanical strength of the material is usually improved by making the skeletons into fully dense. In a specific case of regulating nanoporous monolith in the MTMS compositions, highly light-transmitting low density solids with superb thermal insulation property can be prepared with or without supercritical drying process. ${ }^{27)}$ The regulation of mesopores in the size range between 30 and $50 \mathrm{~nm}$ is conducted by the addition of a surfactant to the starting solution containing only MTMS as a siloxane source. Due to its inherent hydrophobicity, the resultant gel is far more stable against the atmospheric moisture compared with the pure silica counterparts.

\subsection{Double hierarchy}

Since the interconnected macropores enhances the material transport within the bulk gel sample, the exchange of pore liquid with an external solvent can be performed much faster than for the case with gels having only meso- to micropores. Experimentally, the as-gelled wet monolithic specimen is immersed in an excess amount of an external solvent such as aqueous ammonia solution. Alternatively, one can add urea in the starting composition of the gel preparation, and subsequently heat the wet gel in a closed vessel to generate aqueous ammonia in situ. The preferential dissolution of gel network sites with small positive curvature and subsequent reprecipita- tion onto those with small negative curvature results in the reorganization of smaller pores into larger pores (the so called Ostwald ripening mechanism). ${ }^{28)}$ It is noteworthy that gel with much less solubility in external aqueous solutions can also be treated under hydrothermal conditions to give enlarged and well-defined mesopore structures. In any case, these mesopore formation processes take place within the preformed micrometer-sized gel skeletons, so that the size of mesopores can be controlled independently of the macropore size unless the local dissolution of the gel skeletons causes significant deformation of the whole macroporous framework during the solvent exchange.

For the purpose of obtaining mesopores with higher degrees of order in pore size, shape and spatial arrangement, the supramolecular templating is an attractive alternative to the above aging process. It has been found that several kinds of surfactants can be used to induce the phase separation concurrently with the sol-gel transition. ${ }^{29)-32)}$ An appropriate choice of surfactants suitable also to the supramolecular templating of mesopores realized materials with crystal-like long-range ordered mesopores homogeneously located in the micrometersized well-defined gel skeletons. The key to combine the phase-separation/gelation and supramolecular templating/ precipitation is that both processes include a kind of polymerization-induced phase separation. It has been established that cooperative assembly between surfactant micelles and oligomeric oxides enhances the ordered arrangement of the micelles. Highly ordered mesostructure are organized by such cooperative assembly mechanism in generally amorphous oxide networks. Due to relatively strong attractive interactions between micelles and oxides, submicron- to micron-sized particles are precipitated out of the solution in dilute systems under a closed condition.

Starting from a composition favorable for the formation of co-continuous macroporous structure containing a triblock copolymer Pluronic P123 $\left(\mathrm{EO}_{20}-\mathrm{PO}_{70}-\mathrm{EO}_{20}\right.$, EO: ethylene oxide, PO: propylene oxide), an additive, 1,3,5-trimethylbenzene (TMB), known to preferentially distributed to the hydrophobic cores of micelles, was introduced to enhance longrange ordering of mesophases. ${ }^{33), 34)}$ Alternatively, a large amount of water together with high concentration of $\mathrm{P} 123$ was used. The long-range ordering of cylindrical pores in 2-dimensional hexagonal symmetry has been confirmed by the XRD results that indicate sharp peaks comparable to single-crystal like SBA-15. ${ }^{34)}$ Further, the real-space observations have been performed by FE-SEM and TEM. It is noteworthy that the shape of gel skeletons is affected by the anisotropy of mesopores contained in the skeletons; that is, those with cylindrical mesopores exhibit fibrous features.

\subsection{Triple hierarchy}

It is possible to construct additional finer hierarchy in the doubly hierarchical porous solids. By utilizing the so-called template synthesis of zeolitic compounds, precursors to generate silica and alumina are added externally, and allowed to crystallize either in liquid or vapor phase reactions. ${ }^{35), 36)}$ Oxides with well-defined macropores, tailored mesopores and crystal-like micropores can be synthesized in a monolithic form. Through the crystallization process of the zeolitic compounds, the mesopore structure may be modified to some extents, however, the macroporous framework remains almost unaffected. Beads in the size of millimeters with controlled macro-, meso- and microporosities will be useful in the parallel reactors which are recently becoming popular. ${ }^{37)}$ 


\section{Applications and products}

The most successful application of the oxides from sol-gel with phase separation so far is a monolithic HPLC column. In terms of chemical composition, the monolithic columns have been targeted to replace conventional particle-packed columns of high-purity silica. Actually, the monolithic columns modified with $\mathrm{C}_{18}$-ligands perform almost similarly to conventional particle-packed columns with respect to the surface properties, but require dramatically lower column pressure. The monolithic column with 1-micron thick skeleton and 2-micron wide throughpore has the potential to outperform a conventional column packed with 5 -micron spherical particles by an order of magnitude with ordinary instrumentation. For higher performance, theoretically, the reduction is required in the domain size down to sub-micron regime where ordinary instrumentations afford to perform a practical flow rate. However, experimental obstacles exist that lead to locally inhomogeneous structure when the domain size is intended to sub-micron regime. ${ }^{38)}$ The computational fluid dynamics simulation also predicts further improvement of column performance by preparing smaller domains but serious loss by the structural inhomogeneity. ${ }^{39)}$ The optimization of macropore structure requires exact three-dimensional geometrical information of the skeletons and pores, which can now be analyzed by laser scanning confocal microscopy. ${ }^{40)}$ There remains a challenging task to make monolithic columns really state-ofthe-art technology for high-throughput analysis that is highly demanded in pharmatheutical and biochemistry fields.

Monolithic materials with highly controlled inner surfaces such as well-defined hierarchical pores can be used in many of the areas where the particle-packed structure has been playing an important role. The low flow resistance (high permeability) and enhanced accessibility to the nano-scaled surfaces in liquid phase of monolithic hierarchically porous materials are advantageous to every reaction/separation/ purification processes. Liquid-phase catalytic reactions can be enhanced in a device composed of immobilized enzyme or catalyst in general. ${ }^{41)}$ Recently a flow-through bio-reactor made of macroporous silica monolith support incorporated with immobilized trypsin was successfully prepared ${ }^{42)}$ and the pipette-tip type device for rapid digestion by trypsin has also been reported. ${ }^{43)}$ Chromatography in larger dimensions for preparative and process purposes will also benefit by the use of monolithic columns prepared in appropriate module structures. Similarly, materials for solid-phase extraction, SPE, purposes can be easily designed to exhibit superior permeability and extraction efficiency. ${ }^{44)}-46$ )

\section{Conclusions}

Polymerization-induced phase separation in oxide sol-gel systems is unique in that a wide variety of transient multiphase structures are frozen in the gelled samples. Recent success of inducing concurrent phase separation and sol-gel transition in many oxide systems and from various types of precursors implies that the phenomenon is independent of the mechanism of gelation (chemical polymerization or physical aggregation). The very essential requirement is simply 'competitive' phase separation and sol-gel transition both in length scale and in time scale. With an extension of the material shape and size to the smaller dimension, the effect of spatial confinement on the structure development becomes significant. Deeper understanding is still needed to completely control the morphology even in the miniaturized spaces.

Further development of chemical modification of the pore surfaces as well as impregnating functional molecules will enhance the application of the well-defined hierarchically porous material. The successful introduction of supramolecularly templated mesostructures into the well-defined macroporous framework will offer additional possibilities to accommodate molecules, bio-polymers and even living cells complexed with the surfactant mesophases. Continuous efforts are being made to integrate a wide variety of highly-ordered mesophases into well-defined assemblies in longer length scales with extended chemical compositions.

Acknowledgements The author expresses his sincere thanks to all the students who worked with him. Contributions of Profs. Nobuo Tanaka and Hiroshi Jinnai, Kyoto Institute of Technology, and of Prof. Koji Fujita, Kyoto University are gratefully acknowledged. The present work was partly supported by a Grant for Practical Application of University R\&D Results under the Matching Fund Method from New Energy and Industrial Technology Development Organization (NEDO), Japan.

\section{References}

1) Nordberg, M. E., J. Am. Ceram. Soc., Vol. 27, pp. 299-304 (1944).

2) Kaji, H., Nakanishi, K. and Soga, N., J. Sol-Gel Sci. Technol., Vol. 1, pp. 35-46 (1993).

3) Nakanishi, K. and Soga, N., J. Am. Ceram. Soc., Vol. 74, pp. 2518-2530 (1991).

4) Nakanishi, K. and Soga, N., J. Non-Cryst. Solids, Vol. 139, pp. 1-13 (1992).

5) Nakanishi, K., J. Porous Mater., Vol. 4, pp. 67-112 (1997).

6) Konishi, J., Fujita, K., Nakanishi, K. and Hirao, K., Chem. Mat., Vol. 18, pp. 864-866 (2006).

7) Konishi, J., Fujita, K., Nakanishi, K. and Hirao, K., Chem. Mat., Vol. 18, pp. 6069-6074 (2006).

8) Nakanishi, K., Bull. Chem. Soc. Jpn. (Account), Vol. 79, pp. 673-691 (2006).

9) a) Cahn, J. W., Acta Metal., Vol. 9, pp. 795-801 (1961); b) Cahn, J. W., J. Chem. Phys., Vol. 42, pp. 93-99 (1965).

10) Hashimoto, T., Itakura, M. and Hasegawa, H., J. Chem. Phys., Vol. 85, pp. 6118-6128 (1986).

11) a) Tanaka, N., Kobayashi, H., Nakanishi, K., Minakuchi, H. and Ishizuka, N., Anal. Chem., Vol. 73, pp. 420A-429A (2001); b) Minakuchi, H., Nakanishi, K., Soga, N., Ishizuka, N. and Tanaka, N., Anal. Chem., Vol. 68, pp. 3498-3501 (1996).

12) Brinker, C. J. and Scherer, G. W., "Sol-Gel Science; The Physics and Chemistry of Sol-Gel Processing," Academic Press, New York (1990).

13) Flory, P. J., "Principles of Polymer Chemistry," Cornell University Press, Ithaca, New York (1971).

14) deGennes, P. G., "Scaling Concepts in Polymer Physics," Cornell University Press, Ithaca, New York (1979).

15) Nakanishi, K., Komura, H., Takahashi, R. and Soga, N., Bull. Chem. Soc. Jpn., Vol. 67, pp. 1327-1335 (1994).

16) Nakanishi, K. and Kanamori, K., J. Mater. Chem., Vol. 15, pp. 3776-3786 (2005).

17) Nakanishi, K., Yamato, T. and Hirao, K., Mat. Res. Soc. Symp. Proc., Vol. 726, pp. Q9.7.1-10 (2002).

18) Tokudome, Y., Fujita, K., Hirao, K. and Nakanishi, K., partly presented at $19^{\text {th }}$ Fall Meeting of the Ceramic Society of Japan, 19-21 Sept. 2006, Kofu, Yamanashi, Japan.

19) Shoup, R. D., "Colloid and Interface Science," Ed. by Kerker, M., Academic Press, New York (1976) Vol. 3, pp. 63-69.

20) Nishino, H., Takahashi, R., Sato, S. and Sodesawa, T., J. NonCryst. Solids, Vol. 333, pp. 284-290 (2004).

21) Tomita, Y., Takahashi, R., Sato, S., Sodesawa, T. and Otsuda, M., J. Ceram. Soc. Japan, Vol. 112, pp. 491-495 (2004).

22) Takahashi, R., Sato, S., Sodesawa, T. and Azuma, T., J. Sol-Gel Sci. Technol., Vol. 31, pp. 373-376 (2004).

23) Yachi, A., Takahashi, R., Sato, S., Sodesawa, T., Oguma, K., Matsutani, K. and Mikami, N., J. Non-Cryst. Solids, Vol. 351, pp. 331-339 (2005). 
24) Iler, R. K., "The Chemistry of Silica," Wiley, New York (1979) pp. 177.

25) Kanamori, K., Nakanishi, K. and Hanada, T., Adv. Mater., Vol. 18, pp. 2407-2411 (2006).

26) Fuchigami, T., Toki, M. and Nakanishi, K., J. Sol-Gel Sci. Technol., Vol. 19, pp. 337-341 (2000).

27) Kanamori, K., Aizawa, M. and Nakanishi, K., presented at Sol-Gel 2005, Los Angeles, pp. 21-26 August 2005.

28) Nakanishi, K., Takahashi, R., Nagakane, T., Kitayama, K., Koheiya, N., Shikata, H. and Soga, N., J. Sol-Gel Sci. Technol., Vol. 17, pp. 191-210 (2000).

29) Nakanishi, K., Nagakane, T. and Soga, N., J. Porous Mater., Vol. 5, pp. 103-110 (1998).

30) Sato, Y., Nakanishi, K., Hirao, K., Jinnai, H., Shibayama, M., Melnichenko, Y. B. and Wignall, G. D., Colloids and Surfaces A: Physicochemical and Engineering Aspects, Vol. 187/188, pp. 117-122 (2001).

31) Nakanishi, K., Sato, Y., Ruyat, Y. and Hirao, K., J. Sol-Gel Sci. Technol., Vol. 26, pp. 567-570 (2003).

32) Nakanishi, K., Mat. Res. Soc. Symp. Proc., Vol. 788, pp. L7.5.1-10 (2004).

33) Nakanishi, K., Kobayashi, Y., Amatani, T., Hirao, K. and Kodaira, T., Chem. Mat., Vol. 16, pp. 3652-3658 (2005).

34) Amatani, T., Nakanishi, K., Hirao, K. and Kodaira, T., Chem. Mat., Vol. 17, pp. 2114-2119 (2005).

35) Shikata, H., Nakanishi, K. and Hirao, K., J. Sol-Gel Sci.
Technol., Vol. 19, pp. 769-773 (2000).

36) Takahashi, R., Sato, S., Sodesawa, T., Nishino, H. and Yachi, A., J. Ceram. Soc. Japan, Vol. 114, pp. 421-424 (2006).

37) Schüth, F., Busch, O., Hoffmann, C., Johann, T., Kiener, C., Demuth, D., Klein, J., Schunk, S., Strehlau, W. and Zech, T., Topics in Catalysis, Vol. 21, pp. 55-66 (2002).

38) Hara, T., Kobayashi, H., Ikegami, T., Nakanishi, K. and Tanaka, N., Anal. Chem., Vol. 78, pp. 7632-7642 (2006).

39) Gzil, P., Vervoort, N., Baron, V. and Desmet, G., Anal. Chem., Vol. 76, pp. 6707-6718 (2004).

40) Saito, H., Nakanishi, K., Hirao, K. and Jinnai, H., J. Chromatogr. A, Vol. 1119, pp. 95-104 (2006).

41) Takahashi, R., Sato, S., Sodesawa, T., Arai, K. and Yabuki, M., J. Catal., Vol. 229, pp. 24-29 (2005).

42) Kato, M., Inuzuka, K., Sakai-Kato, K. and Toyo'oka, T., Anal. Chem., Vol. 77, pp. 1813-1818 (2005).

43) Ota, S., Miyazaki, S., Matsuoka, H., Morisato, K., Shintani, Y. and Nakanishi, K., J. Biophys., Biochem. Methods, in press.

44) Miyazaki, S., Miah, M. Y., Morisato, K., Shintani, Y., Kuroha, T. and Nakanishi, K., J. Sep. Sci., Vol. 8, pp. 39-44 (2005).

45) Shintani, Y., Zhou, X., Furuno, M., Minakuchi, H. and Nakanishi, K., J. Chromatogr. A, Vol. 985, pp. 351-357 (2003).

46) Miyazaki, S., Morisato, K., Ishizuka, N., Minakuchi, H., Shintani, Y., Furuno, M. and Nakanishi, K., J. Chromatogr. A, Vol. 1043, pp. 19-25 (2004).

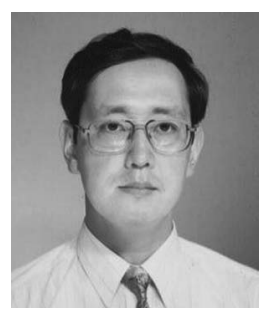

Kazuki Nakanishi is Associate Professor of Department of Chemistry, Graduate School of Science, Kyoto University. After experiencing some research in physical chemistry of polymers at Kyoto University (Masters degree in Industrial Chemistry), he became interested in the competitive processes between phase separation and sol-gel transition in 1986 when he joined Department of Material Chemistry, Graduate School of Engineering, Kyoto University and is still digging into its depth after 20 years. His research focuses on the integrated design of hierarchically porous materials in ceramics, organic-inorganic hybrids and colloidal systems. Awarded: D.R. Ulrich Prize (1997) and ICG Prize in memory of Prof. Vittorio Gottardi (1999). 